\title{
Relative decay conditions on Liouville type theorem for the steady Navier-Stokes system
}

\author{
Dongho Chae \\ Department of Mathematics \\ Chung-Ang University \\ Dongjak-gu Heukseok-ro 84 \\ Seoul 06974, Republic of Korea \\ email:dchae@cau.ac.kr
}

\begin{abstract}
In this paper we prove Liouville type theorem for the stationary Navier-Stokes equations in $\mathbb{R}^{3}$ under the assumptions on the relative decays of velocity, pressure and the head pressure. More precisely, we show that any smooth solution $(u, p)$ of the stationary Navier-Stokes equations satisfying $u(x) \rightarrow 0$ as $|x| \rightarrow+\infty$ and the condition of finite Dirichlet integral $\int_{\mathbb{R}^{3}}|\nabla u|^{2} d x<+\infty$ is trivial, if either $|u| /|Q|=O(1)$ or $|p| /|Q|=O(1)$ as $|x| \rightarrow \infty$, where $|Q|=\frac{1}{2}|u|^{2}+p$ is the head pressure.
\end{abstract}

AMS Subject Classification Number: 35Q30, 76D05, 76D03

keywords: stationary Navier-Stokes equations, Liouville type theorem

\section{Introduction}

We are concerned on the Liouville type problem for the stationary NavierStokes equations in $\mathbb{R}^{3}$.

$$
\left\{\begin{aligned}
u \cdot \nabla u & =-\nabla p+\Delta u \\
\nabla \cdot u & =0
\end{aligned}\right.
$$


Here, $u=\left(u_{1}(x), u_{2}(x), u_{3}(x)\right)$ is a vector field in $\mathbb{R}^{3}$, and $p=p(x)$ is a scalar field. We equip (1.1) with the uniform decay condition at spatial infinity, $|u(x)| \rightarrow 0$ as $|x| \rightarrow+\infty$, which means more precisely

$$
\lim _{R \rightarrow+\infty} \sup _{|x|>R}|u(x)|=0
$$

Obviously $(u, p)$ with $u=0$ and $p=$ constant is a trivial solution to (1.1)(1.2). An important question is if there is other nontrivial solution. This uniqueness problem, or equivalently the Liouville type problem is currently a hot subject of study in the community of mathematical fluid mechanics. In general we impose an additional condition, the finiteness of the Dirichlet integral,

$$
\int_{\mathbb{R}^{3}}|\nabla u|^{2} d x<+\infty
$$

A solution $u$ of the stationary Navier-Stokes equations, satisfying the finite Dirichlet integral condition (1.3), is called D-solution, or Leray solution, following the work due to Leray[11]. The Liouville type problem for D-solution of (1.1)-(1.2) is written explicitly in [6, Remark 9.6, p.147]. After the statement of the problem it is shown that under the assumption $u \in L^{\frac{9}{2}}\left(\mathbb{R}^{3}\right)$ for the solution the solution we have $u=0$. The two dimensional Liouville type problem is solved in [7], while in the $n$-dimensional case with $n \geq 4$, the problem is easily solved by Galdi [6]. Thus, the Liouville type problem for D-solution has been wide open only in the three dimensional case. There are numerous partial results(see e.g. [1, 2, 3, 4, 5, 8, 9, 10, 12, 13, 14, 15 and the references therein), deducing the triviality of solution to (1.1)-(1.3) under various sufficient conditions. Among others the author of this paper obtained in [1] that under the condition together with (1.2) $\int_{\mathbb{R}^{3}}|\Delta u|^{\frac{6}{5}} d x<+\infty$ the solution of (1.1) is trivial, $u=0$ on $\mathbb{R}^{3}$. This is the first condition with the same scaling as (1.3). Another sufficient condition with the same scaling as (1.3) deduced in [2] is

$$
\int_{\mathbb{R}^{3}}|\nabla \sqrt{|Q|}|^{2} d x=\frac{1}{4} \int_{\mathbb{R}^{3}} \frac{|\nabla Q|^{2}}{|Q|} d x<+\infty,
$$

where $Q=\frac{1}{2}|u|^{2}+p$ is the head pressure.

In this paper we prove that the solution of (1.1) is trivial under the conditions (1.2) and (1.3) under the extra assumptions on the relative decay 
assumptions on the velocity, pressure and the head pressure. More precisely we shall prove the following.

Theorem 1.1 Let $(u, p)$ be a smooth solution of (1.1) satifsying the conditions (1.2) and (1.3), and let $Q=\frac{1}{2}|u|^{2}+p$ is its head pressure. Suppose that either

$$
\lim _{R \rightarrow+\infty} \sup _{\{|x|>R\}} \frac{|u|}{|Q|^{\frac{1}{2}}}<+\infty
$$

or

$$
\lim _{R \rightarrow+\infty} \sup _{\{|x|>R\}} \frac{|p|}{|Q|}<+\infty
$$

Then, $u=0$ on $\mathbb{R}^{3}$.

Remark 1.1 Observe that the ratios in (1.4) and (1.5) are scaling invariant. We also note that a lower estimate part of the ratio in (1.5), namely

$$
\lim _{R \rightarrow+\infty} \sup _{\{|x|>R\}} \frac{|p|}{|Q|} \geq 1
$$

holds true. Indeed, from the fact that $Q \leq 0$ on $\mathbb{R}^{3}$ (see (2.6) below), and $p=-\frac{1}{2}|u|^{2}+Q \leq 0$ we find $\frac{|p|}{|Q|}=\frac{1}{|Q|}\left(|Q|+\frac{1}{2}|u|^{2}\right) \geq 1$ on $\mathbb{R}^{3}$, which provides us with (1.6).

\section{Proof of the Theorem}

Proof of Theorem 1.1 Given smooth solution $(u, p)$ of (1.1), we set the head pressure $Q=p+\frac{1}{2}|u|^{2}$ and the vorticity $\omega$ is identified as collection of independent element of the skew-symmetric part of the matrix $\nabla u$, namely $\frac{1}{2} \nabla u-\frac{1}{2}(\nabla u)^{\top}$. Then, it is well-known that the following holds

$$
\Delta Q-u \cdot \nabla Q=|\omega|^{2} .
$$

Indeed, multiplying the first equation of (1.1) by $u$, we have

$$
u \cdot \nabla Q=\Delta \frac{|u|^{2}}{2}-|\nabla u|^{2} .
$$

Taking divergence of (1.1), we are led to

$$
0=\Delta p+\operatorname{Tr}\left(\nabla \mathrm{u}(\nabla \mathrm{u})^{\top}\right) .
$$


Adding (2.3) to (2.2), and observing $|\nabla u|^{2}-\operatorname{Tr}\left(\nabla \mathrm{u}(\nabla \mathrm{u})^{\top}\right)=|\omega|^{2}$, we obtain (2.1). We also recall that it is known (see e.g. Theorem 6.1, p.105, [2]) that the condition (1.2) together with (1.3) implies that there exists a constant $p_{0}$ such that

$$
p(x) \rightarrow p_{0} \quad \text { as } \quad|x| \rightarrow+\infty .
$$

Therefore, redefining $Q-p_{0}$ as a new head pressure, denoted by the same $Q$, we may assume that

$$
Q(x) \rightarrow 0 \quad \text { as } \quad|x| \rightarrow+\infty .
$$

We shall now prove $Q=0$ on $\mathbb{R}^{3}$ by a contradiction argument. Let us suppose on the contrary $Q \neq 0$ on $\mathbb{R}^{3}$. In view of $(2.5)$, applying the maximum principle to (2.1), we observe $Q \leq 0$ on $\mathbb{R}^{3}$. Moreover, by the maximum principle again, either $Q(x)=0$ for all $x \in \mathbb{R}^{3}$, or $Q(x)<0$ for all $x \in \mathbb{R}^{3}$. Indeed, any point $x_{0} \in \mathbb{R}^{3}$ such that $Q\left(x_{0}\right)=0$ is a point of local maximum, which is not allowed unless $Q \equiv 0$ by the maximum principle. Therefore, the assumption $Q \neq 0$ on $\mathbb{R}^{3}$ implies that

$$
Q(x)<0 \quad \forall x \in \mathbb{R}^{3} .
$$

Let us set $m=\sup _{x \in \mathbb{R}^{3}}|Q(x)|>0$. We first claim that

$$
\int_{\{|Q(x)|>\lambda\}} u \cdot \nabla Q f(|Q|) d x=0
$$

for any continuous function $f$ on $\mathbb{R}$, and for almost every $\lambda \in[0, m]$. Indeed,

$$
\begin{aligned}
\int_{\{|Q(x)|>\lambda\}} & u \cdot \nabla Q f(|Q|) d x=-\int_{\{|Q(x)|>\lambda\}} u \cdot \nabla\left(\int_{0}^{|Q(x)|} f(s) d s\right) d x \\
& =-\int_{\{|Q(x)|=\lambda\}} u \cdot \nu\left(\int_{0}^{|Q(x)|} f(s) d s\right) d S \\
& =-\left(\int_{0}^{\lambda} f(s) d s\right) \int_{\{|Q(x)|=\lambda\}} u \cdot \nu d S \\
& =-\left(\int_{0}^{\lambda} f(s) d s\right) \int_{\{|Q(x)|>\lambda\}} \nabla \cdot u d x=0
\end{aligned}
$$

where $\nu$ denotes the outward unit normal vector on the boundary of the domain

$$
\Omega_{\lambda}:=\left\{x \in \mathbb{R}^{3}|| Q(x) \mid>\lambda\right\},
$$


and we used Sard's theorem to justify the use the divergence theorem on the domain $\Omega_{\lambda}$, whose boundary $\partial \Omega_{\lambda}$, is a smooth level surface for each $\lambda \in(0, m)$ expect possibly measure zero set of points. Integrating (2.7) over $\Omega_{\lambda}$, and using (2.7) for $f(s) \equiv 1$, we are led to

$$
\int_{\{|Q(x)|>\lambda\}} \Delta Q=\int_{\{|Q(x)|=\lambda\}}|\nabla Q(x)| d S=\int_{\{|Q(x)|>\lambda\}}|\omega|^{2} d x .
$$

Given $\theta \in(0,1)$ we introduce a smooth, non-decreasing cut-off function $\eta=\eta_{\lambda} ;[0, \infty) \rightarrow[0,1]$ by

$$
\eta(s)= \begin{cases}0 & \text { if } \quad 0 \leq s<1-\theta, \\ 1 & \text { if } \quad s \geq 1\end{cases}
$$

together with $0 \leq \eta^{\prime}(s) \leq \frac{2}{\theta}$ for all $s \in(0, \infty)$. Then, we set

$$
\varphi_{\lambda}(x)=\eta\left(\log \left(\frac{|Q(x)|}{\lambda}\right)\right) .
$$

Note that $\varphi \in C_{c}^{\infty}\left(\mathbb{R}^{3}\right)$ for almost every $\lambda \in\left[0, \frac{m}{e^{2}}\right)$, and

$$
\varphi_{\lambda}(x)= \begin{cases}0 & \text { if } \quad 0<|Q(x)|<\lambda e^{1-\theta}, \\ 1 & \text { if } \quad|Q(x)| \geq \lambda e\end{cases}
$$

and therefore, $\varphi_{\lambda}(x) \rightarrow 1$ as $\lambda \rightarrow 0$ for almost everywhere in $\mathbb{R}^{3}$. We claim that for $\theta \in(0,1)$

$$
\int_{\{\theta \lambda<|Q(x)|<\lambda\}} \frac{|\nabla Q|^{2}}{|Q|} d x \leq\left(\log \frac{1}{\theta}\right) \int_{\{|Q(x)|>\theta \lambda\}}|\omega|^{2} d x .
$$

Indeed, using the co-area formula and (2.9), we estimate

$$
\begin{aligned}
\int_{\{\theta \lambda<|Q(x)|<\lambda\}} \frac{|\nabla Q|^{2}}{|Q|} d x=\int_{\theta \lambda}^{\lambda} \frac{1}{s}\left(\int_{\{|Q(x)|=s\}}|\nabla Q(x)| d S\right) d s \\
=\int_{\theta \lambda}^{\lambda} \frac{1}{s}\left(\int_{\{|Q(x)|>s\}}|\omega|^{2} d x\right) d s \leq \int_{\theta \lambda}^{\lambda} \frac{d s}{s} \int_{\{|Q(x)|>\theta \lambda\}}|\omega|^{2} d x \\
=\left(\log \frac{1}{\theta}\right) \int_{\{|Q(x)|>\theta \lambda\}}|\omega|^{2} d x,
\end{aligned}
$$


as claimed. We observe that this implies

$$
\int_{\left\{e^{1-\theta} \lambda<|Q(x)|<e^{\lambda}\right\}} \frac{|\nabla Q|^{2}}{|Q|} d x \leq \theta \int_{\left\{|Q(x)|>e^{1-\theta} \lambda\right\}}|\omega|^{2} d x .
$$

Multiplying (1.1) by $u$, we find

$$
\Delta|u|^{2}-2(u \cdot \nabla) Q=2|\nabla u|^{2} .
$$

Next, multiplying (2.16) by $\varphi_{\lambda}(x)$, integrating it over $\mathbb{R}^{3}$, and integrating by part, we obtain

$$
\begin{aligned}
\int_{\mathbb{R}^{3}}|\nabla u|^{2} \varphi_{\lambda} d x= & -\frac{1}{2} \int_{\mathbb{R}^{3}} \frac{\nabla|u|^{2} \cdot \nabla Q}{|Q|} \eta^{\prime}\left(\log \left(\frac{|Q(x)|}{\lambda}\right)\right) d x \\
& -\int_{\left\{|Q(x)|>e^{1-\theta} \lambda\right\}}(u \cdot \nabla) Q \varphi_{\lambda} d x \\
= & I_{1}+I_{2} .
\end{aligned}
$$

We assume now (1.4). From (2.7) it follows immediately that $I_{2}=0$. Using (2.15), we estimate $I_{1}$ as follows

$$
\begin{aligned}
I_{1} & \leq \frac{2}{\theta} \int_{\left\{e^{(1-\theta)} \lambda<|Q(x)|<e \lambda\right\}} \frac{|u|}{|Q|^{\frac{1}{2}}} \frac{|\nabla Q|}{|Q|^{\frac{1}{2}}}|\nabla u| d x \\
\leq & \frac{2}{\theta} \sup _{\left\{e^{(1-\theta)} \lambda<|Q(x)|<e \lambda\right\}} \frac{|u|}{|Q|^{\frac{1}{2}}}\left(\int_{\left\{e^{(1-\theta)} \lambda<|Q(x)|<e \lambda\right\}} \frac{|\nabla Q|^{2}}{|Q|} d x\right)^{\frac{1}{2}} \times \\
& \times\left(\int_{\left\{e^{(1-\theta)} \lambda<|Q(x)|<e \lambda\right\}}|\nabla u|^{2} d x\right)^{\frac{1}{2}} \\
\leq & \frac{2}{\sqrt{\theta}} \sup _{\left\{e^{(1-\theta)} \lambda<|Q(x)|<e \lambda\right\}} \frac{|u|}{|Q|^{\frac{1}{2}}}\left(\int_{\left\{|Q(x)|>e^{(1-\theta)} \lambda\right\}}|\omega|^{2} d x\right)^{\frac{1}{2}} \times \\
& \times\left(\int_{\left\{e^{(1-\theta)} \lambda<|Q(x)|<e \lambda\right\}}|\nabla u|^{2} d x\right)^{\frac{1}{2}} .
\end{aligned}
$$

We fix $\theta \in(0,1)$. Let $B_{R}=\left\{x \in \mathbb{R}^{3}|| x \mid<R\right\}$. From (2.5) we find that for any $R>0$ there exists $\lambda=\lambda_{R}$ such that

$$
B_{R} \subset\left\{x \in \mathbb{R}^{3}|| Q(x) \mid>e \lambda\right\} \subset\left\{x \in \mathbb{R}^{3}|| Q(x) \mid>e^{1-\theta} \lambda\right\},
$$


and

$$
\lambda_{R} \rightarrow 0 \quad \text { as } \quad R \rightarrow+\infty .
$$

From this observation we have from (2.17) and (2.18), replacing $\lambda$ by $\lambda_{R}$, that

$$
\int_{B_{R}}|\nabla u|^{2} d x \leq \frac{2}{\sqrt{\theta}} \sup _{\{|x|>R\}} \frac{|u|}{|Q|^{\frac{1}{2}}}\left(\int_{\mathbb{R}^{3}}|\omega|^{2} d x\right)^{\frac{1}{2}}\left(\int_{\{|x|>R\}}|\nabla u|^{2} d x\right)^{\frac{1}{2}} .
$$

Passing $R \rightarrow \infty$ on the both sides of (2.19), and applying the Lebesgue dominated convergence theorem, we obtain $\int_{\mathbb{R}^{3}}|\nabla u|^{2} d x=0$, and $u=$ constant on $\mathbb{R}^{3}$. Combing this with the boundary condition (1.2), we find $u=0$ on $\mathbb{R}^{3}$. Regarding the condition (1.5), we just observe

$$
\left(\frac{|u|}{|Q|^{\frac{1}{2}}}\right)^{2} \leq \frac{2|Q|+2|p|}{|Q|}=2+2 \frac{|p|}{|Q|}
$$

and

$$
\frac{|p|}{|Q|} \leq \frac{|Q|+\frac{|u|^{2}}{2}}{|Q|} \leq 1+\frac{1}{2}\left(\frac{|u|}{|Q|}\right)^{2}
$$

Hence, the conditions (1.4) and (1.5) are equivalent to each other.

\section{Acknowledgements}

This research was supported partially by NRF grants 2016R1A2B3011647.

\section{References}

[1] D. Chae, Liouville-type theorem for the forced Euler equations and the Navier-Stokes equations. Commun. Math. Phys., 326, pp. 37-48, (2014).

[2] D. Chae, Note on the Liouville type problem for the stationary NavierStokes equations in $\mathbb{R}^{3}$ J. Diff. Eqns, 268, no. 3,pp. 1043-1049, (2020)

[3] D. Chae and J. Wolf, On Liouville type theorem for the stationary NavierStokes equations, Calc. Var. PDE, 58, no. 3 (2019), Art. 111, (2019). 
[4] D. Chae and T. Yoneda, On the Liouville theorem for the stationary Navier-Stokes equations in a critical space, J. Math. Anal. Appl. 405, no. 2, 706-710, (2013).

[5] D. Chamorro, O. Jarrin and P- G. Lemarié-Rieusset, Some Liouville theorems for stationary Navier-Stokes equations in Lebesgue and Morrey spaces, C.R. Math. Acad. Sci. Paris. 357, no. 2, pp. 175-179, (2019).

[6] G. P. Galdi, An introduction to the mathematical theory of the NavierStokes equations, Volume II (Nonlinear Steady Problems), Springer, New York, (1994).

[7] D. Gilbarg and H. F. Weinberger, Asymptotic properties of steady plane solutions of the Navier-Stokes equations with bounded Dirichlet integral, Ann. Sc. Norm. Super. Pisa, 4, no. 5, pp. 381-404, (1978).

[8] G. Koch, N. Nadirashvili, G. Seregin, V. Šverék, Liouville theorems for the Navier-Stokes equations and applications, Acta Mathematica, 203, pp. 83-105, (2009).

[9] M. Korobkov, P. Pileckas, R. Russo, The Liouville theorem for the steadystate NavierStokes problem for axially symmetric 3D solutions in absence of swirl, J. Math. Fluid Mech. 17, no. 2, pp. 287-293, (2015).

[10] H. Kozono, Y. Terasawa, Y. Wakasugi, A remark on Liouville-type theorems for the stationary Navier-Stokes equations in three space dimensions, J. Func. Anal, 272, pp. 804-818, (2017).

[11] J. Leray, Étude de diverses équations intégrales non linéaire et de quelques problémes que pose l'hydrodynamique, J. Math. Pures Appl. 12, pp. 1-82, (1933).

[12] W. Lyu and S. Weng, Decay rates of second order derivatives of axisymmetric D-solutions to the stationary Navier-Stokes equations, J. Diff. Eqns, in press, https://doi.org/10.1016/j.jde.2020.04.012.

[13] G. Seregin, Liouville type theorem for stationary Navier-Stokes equations, Nonlinearity, 29, pp. 2191-2195, (2016),

[14] G. Seregin, Remarks on Liouville type theorems for steady-state NavierStokes equations, Algebra \& Analysis, 30, no.2, pp. 238-248, (2018). 
[15] G. Seregin and W. Wang, Sufficient conditions on Liouville type theorems for the 3D steady Navier-Stokes equations, Algebra \& Analysis, 31, no. 2, pp. 269-278, (2019). 